\title{
EL PENSAMIENTO DE LAMARCK EN SU CONTEXTO HISTÓRICO
}

\author{
Faustino Cordón \\ Fundación para la Investigación sobre la Biología Evolutiva (FIBE) - Avda. de América 18, Madrid
}

LOS ANTECEDENTES HISTÓRICOS Y LA CIRCUNSTANCIA DE LAMARCK (1744-1829).

Desde mi punto de vista de biólogo interesado en el planteamiento actual de la evolución, no de historiador de la ciencia, se me impone que para enjuiciar con conocimiento de causa el punto de partida y el progreso que supone la obra de Lamarck - que vive de 1744 a 1829- hay que hacer una recapitulación, por sucinta que sea, del orden de conceptos y de problemas con que se enfrenta la ciencia de su época y, en particular, las ciencias naturales, que se ocupan de cómo se ofrece directamente la Naturaleza al hombre. Es obvio que la Naturaleza es captada por los hombres mediante sus órganos de los sentidos, modelados, en todas y cada una de las especies animales, para distinguir y actuar frente a los animales y plantas que constituyan el objeto principal de la actividad de los individuos de cada una, y para desplazarse por el entorno - estructurado por la interferencia de ámbitos de la materia en sus tres estados- que constituye el escenario, asimismo específico, de la vida de todo animal. De este modo, por la índole animal del hombre (el animal racional), se entiende que la ciencia, desde su fase empírica, diferenciara "los tres reinos de la Naturaleza", por características que le parecen inequívocas. Por una parte, los reinos animal y vegetal parecen tener en común el constar de individuos (unidades) que se reproducen a lo largo de las generaciones en hijos iguales a los padres con lo que, en uno y otro reino, se distinguen netamente especies, cuyos ejemplares se caracterizan por una configuración, un tamaño y un comportamiento específicos; individuos que existen durante un tiempo (el transcurso de su vida durante la que crecen, cambian de un modo y con un tempo asimismo específicos) y que muriendo se reducen a lo inorgánico. Se entiende que a los naturalistas del siglo XVIII el reino mineral se les presentara con características que parecían las contrarias a las que les ofrecían en común los animales y las plantas; a saber: el reino mineral parece constar de substancias, de algo continuo (las diversas materias sólidas, el agua, el aire) donde no se distinguen individuos (unidades), ni transcurso definidor de existencia, pero sí, en cambio, se 


\section{FAUSTINO CORDÓN}

definidor de existencia, pero sí, en cambio, se conocen transformaciones (en ocasiones reversibles por la actividad humana) de unas substancias en otras.

Conforme a ese contraste de características entre, por una parte, los dos reinos animal y vegetal y, por otra, el reino mineral, se entiende que los científicos ocupados en el estudio de los dos primeros reinos (los zoólogos y los botánicos) hubieron de plantearse en dicho siglo un orden de tareas basado en unos conceptos y siguiendo unos métodos radicalmente distintos que el orden de temas, conceptos y métodos de los estudiosos del reino mineral (los geólogos y los químicos). Paradójicamente, los resultados obtenidos por unos y por otros condujeron a resultados, en cierto modo, confluentes.

Se entiende que los zoólogos y los botánicos, en posesión del concepto objetivo, verdadero, de especie (todo animal o planta pertenece a una especie que no cambia a escala de la memoria histórica humana ${ }^{1}$ ) y enfrentados con la riqueza de las especies que fue mostrada en su amplitud potencial por los grandes viajes geográficos, se vieran incitados a la difícil tarea (que ocupó a gran número de naturalistas del siglo XVIII, que pueden personificarse en Linneo (1707-1778), en los botánicos franceses De Jussieu y, en fin, en Lamarck mismo) de dominar empíricamente esta gran diversidad, mediante una difícil clasificación adecuada por criterios que parecía que habían de ser artificiales y aceptados o rechazados por su valor pragmático (dado que las especies se suponía tradicionalmente resultados de actos de creación independiente). Por ejemplo, teniendo en cuenta que en el reino vegetal - como en el animal- todas las especies se reproducen y que la flor y el fruto que de ella deriva son órganos de la reproducción, los caracteres florales constituyeron para Linneo un importante criterio de clasificación para un gran conjunto de especies de plantas, etc. Pero el hecho notable que hay que destacar aquí es que las analogías y las diferencias entre las especies animales impusieron a los zoólogos una única clasificación viable de ellas, el sistema natural, en el que los caracteres utilizados para incluir una especie en una categoría dada no son sólo los propios de esta categoría, sino, como regla general, la suma de los privativos de la categoría y de los de cada una de las categorías superiores; por ejemplo, el lobo necesariamente suma a sus caracteres específicos los caracteres de la familia Canidae a la que pertenece su especie, los del orden Carnívora al que pertenece tal familia, los de la clase Mammalia al que pertenece el orden y en fin los del filum Chordata al que pertenece la clase Mammalia. De este modo, la clasificación de las especies (cada una constituida por individuos iguales entre sí, que se suponía procedentes de una pareja originaria resultado de un acto de creación independiente) demostró la conclusión inesperada - contradictoria con el supuesto del origen independiente de cada especie animal- de que el concies

I Al parecer, Linneo aplicó el aforismo Natura non facit saltus para señalar la fijeza de las espe-

Asclepio-Vol. XLVIII-I-1996 


\section{EL PENSAMIENTO DE LAMARCK EN SU CONTEXTO HISTÓRICO}

junto de ellas mostraba una unitariedad antes impensable, a saber, se dejaba clasificar en un sistema objetivo - sin otro alternativo imaginable - en el que cada especie que se descubría encontraba su lugar inequívoco por las analogías y diferencias que mostraba con todas y cada una de las restantes; capacidad de ser clasificados que sólo puede convenir a seres (en este caso los animales de todas las especies) que tengan comunidad de origen y que se hayan diferenciado en un proceso conjunto de evolución gradual.

Consideremos ahora el proceso que, a lo largo del siglo XVIII, mostró la ciencia de lo inorgánico, en concreto la química. En contraste con los zoólogos, los químicos comenzaron enfrentándose no con unidades definidas, sino con substancias que no mostraban existencia ni tamaño específicos, ni se reproducían, sino que se mezclaban y se transformaban unas en otras, de un modo, que, a veces, aprendía a realizar o a revertir el hombre. Sobre la base de la tradición empírica humana ganada, con resultados prácticos, en la transformación de lo inorgánico y de la suma de conocimientos obtenida por los alquimistas, los químicos emprendieron tareas científicas como son: resolver una mezcla de substancias en las substancias que la componen (un ejemplo destacado, el aire en sus componentes); transformar unas substancias en otras; procurar interpretar procesos como la combustión, el efecto del agua sobre algunas substancias, el desarrollo de las primeras industrias químicas, etc. Aquí hemos de limitarnos a señalar que los esfuerzos tanteantes de los químicos de los siglos XVII y XVIII sientan la base del trabajo de Lavoisier (1743-1794) que establece la teoría de la química moderna: explica la combustión, establece la composición del agua, el papel del agua y del oxígeno del aire en la formación de ácidos y de bases, distingue entre substancias simples (de las que conoce 23) y compuestas, establece, con el ejemplo de experimentos sencillos e impecables, el método experimental propio de esta ciencia y culmina con el descubrimiento de la ley de la conservación de la materia (1785). En nuestro contexto hay que destacar que Lavoisier desencadenó un proceso de acelerado desarrollo de la química cuyo esencial despliegue se produce a lo largo del siglo XIX y que, ya en 1804 - a sólo diez años de la muerte de Lavoisier-, conduce a la enunciación por Dalton de la teoría atómica, esto es, de que las substancias, tanto simples como compuestas, constan de unidades (los átomos) supuestas eternas y que, de hecho, son intransformables en las reacciones químicas. Como es de conocimiento general, estas unidades se caracterizan (aparte de sus relaciones en las substancias tanto simples como compuestas) por el peso relativo - primero establecidodel átomo de cada especie con respecto al de uno de ellos tomado como unidad $y$, luego, al peso absoluto mismo, determinado por muy diversos métodos con resultados concordantes cuya ínfima magnitud se expresa por el número de Avogadro.

Sin entrar en la interpretación moderna de la estructura, compuesta, del átomo, recordemos que, en 1861, los químicos admitieron, como verdad confirmada, la existencia de unidades materiales del nivel de integración directamente supratómico, las 


\section{FAUSTINO CORDÓN}

moléculas. A unidades de este nivel está elevada toda la materia inorgánica de las capas externas de la Tierra, y los mismos seres vivos constan ponderalmente de moléculas. La diferenciación de las moléculas como unidades de nivel directamente supratómico concretó a los químicos el objetivo de su estudio, a saber, la estructura, en átomos, de las moléculas, las propiedades de cada especie de moléculas, las leyes que se descubren en las transformaciones de unas en otras (i.e., las reacciones químicas), etc... Lo que aquí interesa destacar es que, en tiempos de Lamarck, se produjeron sendas inflexiones, en cierto modo de signo contrario, en la consideración científica de los animales en la de lo inorgánico. La zoología cuya ocupación principal venía siendo la clasificación, por criterios supuestamente artificiales, de las especies de animales, cuya neta diferenciación y cuya estabilidad tienen una base objetiva que se impone a nuestros sentidos y que justificaba el aserto de proceder de remotos actos de creación independiente, terminó estableciendo el sistema natural de las especies que acogía sin distorsión las que se iban descubriendo y que relacionaba cada una con el conjunto de todas, de modo tan coordinado que imponía la existencia de algún tipo de relaciones entre ellas, cuyo intento de interpretación había de irse planteando a los zoólogos. En cambio, la química recorre un camino, en cierto sentido, inverso; parte de substancias que encubren su carácter discontinuo a los sentidos a los que, en cambio, les muestran la comunidad de naturaleza que las permite mezclarse y transformarse unas en otras; y, a partir del estudio empírico de esta conducta recíproca, la química llega a la conclusión -inicialmente impredecible pero que le es impuesta por leyes generales que reveló el análisis comparado de las transformaciones-de que todas las substancias constan de un centenar de especies distintas pero ponderables, los llamados átomos, a las que se comienza considerando simples y eternas - de hecho, son inmutables en todas las reacciones químicas, que transforman unas substancias en otras ${ }^{2}$ - de cuyas combinaciones resultan unidades de nivel directamente supratómico, las moléculas, cuyas propiedades remiten a los átomos que las constituyen y al modo recíproco de estar situados en ellas; los químicos han estudiado gran número de especies de moléculas naturales (tanto inorgánicas como, en mucho mayor número, moléculas carbonadas producidas en el metabolismo intracelular) y sintetizado y analizado en número aún mucho mayor de moléculas artificiales.

Pero, además, entre el despliegue de la zoología y el de la química que hubo de vivir Lamarck, se observa una diferencia en la índole de conceptos, problemas y métodos que dominan en una otra ciencia. La zoología parte de un orden de conceptos, problemas y métodos empíricos (la ciencia empírica exige describir, clasificar y reproducir, lo que se ve); como tarea principal, esta ciencia se ocupó, desde 1750, en

2 Como es de conocimiento general, su descubridor (en 1804) Dalton los identificó con los átomos, de diversa forma, que - según la teoría de Demócrito - son los constituyentes de la materia universal. 


\section{EL PENSAMIENTO DE LAMARCK EN SU CONTEXTO HISTÓRICO}

describir, clasificar las especies de animales conforme a sus caracteres visibles y llegó, como se ha señalado, a una unitariedad del conjunto de ellas, cuya interpretación plantea un problema real, objetivo, pero irresoluble para la época por la ausencia total de datos experimentales concernientes. En tanto que la química parte de una base corta de datos empíricos y se eleva rápidamente con Lavoisier a ciencia experimental que va induciendo leyes que permiten predecir reacciones entre moléculas con la ayuda de un pensamiento teórico cada vez más coherente. Este desarrollo experimental, que permite predecir el resultado de procesos invisibles, confirió a la química, como a otras ciencias de lo inorgánico, un prestigio que hizo que el saber científico se confundiera con entender en términos de lo inorgánico ${ }^{3}$ lo que ejerció sobre el desarrollo posterior de la biología la frecuente tendencia extraviada a intentar explicar los seres vivos en términos de sus moléculas. Así Lamarck remite la explicación de los seres vivos a procesos mecánicos en defensa de la recusación del dualismo entre espíritu y materia, leit motiv del vigoroso enciclopedismo francés del siglo XVIII.

\section{LA APORTACIÓN DE LAMARCK A LA BIOLOGÍA.}

1) El mérito principal de Lamarck es su recusación de la idea dominante de que las especies de animales deban su origen a sendas creaciones de parejas de animales de cada especie que, desde entonces, vienen reproduciéndose con ligeras variaciones individuales que se mantienen de generación en generación y que sólo son imputables a la imperfección relativa de la reproducción; según esta interpretación tradicional, toda especie se ha mantenido invariable desde el acto originario de su creación; el creacionismo de las especies se ha mantenido por pruebas, al parecer firmes, como son: la existencia real de especies animales; su aparente estabilidad a escala no ya de las vidas humanas, sino de la memoria social; y, en fin, las diferencias cualitativas que, frente a lo inorgánico, caracterizan a los seres vivos, a saber, su

\footnotetext{
3 De hecho, todas las ciencias de lo inorgánico (las distintas ramas de la mecánica, el estudio de los fenómenos térmicos, luminosos, electromagnéticos, etc.) como las ciencias biológicas, por la naturaleza molecular a que se ha elevado la realidad en todo el ámbito terrestre que alberga la vida, han modelado los órganos de los sentidos animales y los sistemas musculares adecuándolos a la percepción y manejo de substratos de nivel molecular, de modo que el hombre, como los demás animales, opera, siempre, por mediación de la realidad elevada a nivel molecular y configurada natural o artificialmente por ella en estado sólido. Ahora bien, las moléculas son unidades directamente supratómicas (y directamente infrabasibiónicas —infraproteínicas-) esencialmente homogéneas entre ellas mismas, pero esencialmente heterogéneas con las unidades de los niveles gradualmente inferiores (átomos, partículas nucleares, electrones, fotones) y con las de los superiores (basibiones, células, animales) para las que rigen leyes distintas, privativas para las de cada nivel.
} 
capacidad de tomar noticia, la de actuar tras fines individuales, la de reproducirse, la complejidad interna de los cuerpos orgánicos y el perfecto coajuste de sus partes; características que hacían difícil, en el nivel empírico del conocimiento, reducir a una comunidad de origen lo vivo y lo inorgánico ni sospechar la posibilidad de un proceso inteligible ancestral del que, a partir de lo inorgánico, resultaran los primeros seres vivos. En tal estado de la opinión dominante, útil para los taxonomistas, el creacionismo se iba conmoviendo por el despliegue de la ciencia experimental en diversos campos del conocimiento y por la crítica de los enciclopedistas, una y otros reacios a recurrir a lo suprasensible para explicar los seres, fenómenos y procesos reales, de lo que constituyen testimonios diversas reacciones anticreacionistas contemporáneas de Lamarck ${ }^{4}$. Como recusación frontal al creacionismo de las especies animales, Lamarck emite su teoría innovadora de que los animales más sencillos (él dice, infusorios o pólipos) surgen continuamente de lo inorgánico por generación espontánea, de modo que cada surgimiento inicia una línea de descendencia en la que, a través de cambios insensibles y a lo largo del tiempo, se van sucediendo, sin solución de continuidad, especies cada vez más perfeccionadas hasta culminar en el hombre (despliegue evolutivo de la scala naturae). Lamarck remite la causa de la evolución progresiva de los animales a su tendencia a realizar cada vez mejor su conducta instintiva, lo que influye sobre la configuración somática ("la función crea el órgano") y postulando que los caracteres adquiridos se heredan (la capacidad de reproducirse es una cualidad distintiva entre los seres vivos y lo inorgánico, con la que cuenta certeramente Lamarck). Lamarck no descarta influencias secundarias del ambiente inorgánico sobre la evolución de algunas especies.

Tal es la teoría de la evolución de Lamarck que, en lo esencial, sustituye el creacionismo teológico por un primer planteamiento científico de la diversidad de los animales, avance que podemos puntualizar así: considerar la diversidad de los animales como objeto potencial de conocimiento científico, en cuanto resultado de una evolución determinada; considerar el animal como agente de la propia evolución; considerar los cambios somáticos como efecto de la actividad de los seres vivos y que los caracteres adquiridos se heredan, lo que básicamente, de un modo u otro, parece verdad ya que todo animal en sus fases embrionaria y fetal adquiere caracteres heredados (que en fase correspondiente adquirió cada uno de los padres) y es dado a luz con la capacidad congénita de ir adquiriendo los que le impone su conducta específica sumamente determinada (salvo en el caso del hombre). Pasando por alto sus errores y sus limitaciones de época, en lo dicho hemos procurado destacar el aspecto positivo de la concepción evolucionista de Lamarck.

\footnotetext{
4 Como figuras destacadas que, de diversas formas, tantean reacciones al creacionismo tradicional pueden citarse a Kant (1724-1804), Goethe (1749-1832) y Geoffroy Saint-Hilaire (1772-1844).
} 
Hay que señalar que Lamarck, aparte de su mérito principal, de haberse planteado y abordado por primera vez el proceso indudable de la evolución biológica, y de haber trabajado con aplicación y originalidad en taxonomía de los vegetales, a lo que debió su status académico, fue un taxonomista zoológico eminente al que se deben aportaciones certeras y cruciales como las siguientes:

2) Lamarck desarrolló la clasificación de los animales inferiores (de los por él denominados invertebrados) descuidada por Linneo y continuadores; intuyó certeramente (por asociación de ideas con su pensamiento evolucionista) que en los animales inferiores se dan criterios de agrupación y de diferenciación taxonómicos principales, más básicos, aunque también más recónditos (más difíciles de definir e interpretar), que los que se dan en las categorías subordinadas de ellos y en los animales superiores.

3) Se plantea conscientemente la necesidad de ir estableciendo una clasificación natural, frente al mare magnum de clasificaciones artificiales, sugeridas por criterios parciales y pragmáticos de los naturalistas; esto es, Lamarck persigue explícitamente una clasificación que procure reflejar el proceso por el que "la Naturaleza fue diferenciando los animales", esto es, la evolución misma; para conseguirlo recomienda atender en lo posible a caracteres estructurales internos,en vez de a diferencias externas, más fácilmente perceptibles pero menos básicas que las estructurales.

4) Opina Lamarck, lúcidamente, que una clasificación racional de los animales, para ser conforme con la marcha de la evolución, ha de proceder de abajo arriba, esto es, comenzar con los animales inferiores -infusorios o pólipos, según él- y terminar con el hombre, conforme al orden ascendente de su scala naturae y no a la historia - ajena a ellas - de haberlas ido conociendo y describiendo el hombre; conforme a esta inducción emprendió, con continuidad y clarividencia, la clasificación de los invertebrados, hasta entonces descuidada, en los que estableció categorías taxonómicas principales que siguen intangibles.

5) Lamarck prestó mucha atención a los fósiles de invertebrados, buscando las analogías entre formas muy antiguas y las actuales con el propósito no de datar el origen de éstas - su antiguiedad filogénica, fuera de su orden de ideas-, sino, ante todo, de inquirir las transformaciones geológicas de océanos y de continentes que él considera determinantes de caracteres secundarios de la evolución biológica.

6) Enuncia claramente la ascendencia animal del hombre, al que, anticipándose más de sesenta años a Darwin —aunque del modo, más impreciso, que corresponde a la diferencia de sus respectivas interpretaciones de la evolución-, considera procedente de un mono superior por obvias razones de anatomía comparada. 


\section{FAUSTINO CORDÓN}

\section{LAS CONTRADICCIONES INTERNAS QUE SE ESCONDEN EN LA INTERPRETACIÓN LAMARCKIANA DE LA EVOLUCIÓN ANIMAL.}

Parece inobjetable la aseveración de Lamarck de que entre toda especie y la anterior de la que ella directamente procede no puede, en ningún momento previo a la diferenciación, haberse establecido una solución de continuidad que las distinga (Natura no facit saltus) $)^{5}$. Y, sin embargo, es un hecho inobjetable que entre todo par de especies actuales, por próximas que sean, hay netas soluciones de continuidad. Son dos asertos ambos verdaderos por lo que para un científico - por decirlo así, para un profesional del monismo- no pueden ser sino aparentemente antitéticas y ello incitarle a buscar una interpretación que dé cuenta de la una por la otra y viceversa; claro que la interpretación capaz de resolver la antinomia estaba fuera del alcance de la época de Lamarck, lo que le llevó a dar por resuelta la antinomia por el modo expeditivo de desmentir el término de ella que le pareció que guardaba un sabor creacionista, a saber, la discontinuidad entre especies, esto es, la existencia objetiva, real, de las especies, que había merecido la confirmación de más de medio siglo de trabajo de imnumerables naturalistas, y a cuya clasificación, paradójicamente, él había contribuido de modo importante.

En cambio, Darwin dio un gran paso hacia la interpretación de la evolución de los animales sin sacrificar ninguno de los términos de la aparente antinomia con sus dos aportaciones esenciales: a) con su teoría, con la firme base experimental de las razas domésticas, de que toda especie evoluciona progresivamente por la selección natural como progenitores de los individuos más aptos; y b) al postular que la evolución así cumplida de toda especie culmina en la diferenciación de ella en dos. Es cierto que Darwin no explicó cómo se verifica este proceso de la especiación, lo que no puede hacerse sin entender en qué consiste el medio selector de cada especie y cómo él,

5 Entre todo individuo de toda especie animal y los padres hay siempre diferencias pequeñas (la reproducción animal es imperfecta), pero diferencias que no afectan en el mismo sentido a los individuos de toda la especie cuyo valor promedio de todos sus caracteres y capacidades permanece sensiblemente constante de generación en generación. Esto es lo que se significa, en este contexto, por Natura non facit saltus, que para Linneo constituía un argumento en favor de la naturaleza inmutable de cada una de las especies creadas. Ahora bien, se entiende que un cambio dirigido, común, producido en el ambiente de un conjunto de seres vivos de una especie y que se prolongase a lo largo de muchas generaciones es lo que pudo terminar diferenciando el grupo en una especie nueva, distinta del resto de los enfrentados con el ambiente ancestral. En términos concretos, el problema es cómo pueda producirse esto y cómo de ello resulten no dos razas o variedades, sino dos especies. Contestar este problema (que plantea, sin abordarlo, Darwin) significaría explicar cómo se verifica el proceso de la bifurcación de una especie, la especiación. Digamos de pasada que Darwin no se enfrentó con un caso real de especiación en la diferenciación entre el pinzón continental y el que fue llevado a las Islas Galápagos (caso pertinente a la diferenciación de faunas), sino en las diferenciaciones simpátridas de estos pájaros desde su asentamiento en las Islas Galápagos. 


\section{EL PENSAMIENTO DE LAMARCK EN SU CONTEXTO HISTÓRICO}

como condición previa de la especiación, ha de bifurcarse previamente en dos medios selectores; cuestiones que plantea tácitamente Darwin, pero que no enuncia ni, menos, aborda.

Pasemos a otro grave extravío de la concepción lamarckiana de la evolución de los animales que, al parecer sin hacerse cuestión de ella, rectifica también Darwin. En su apasionada recusación del creacionismo, Lamarck, sin datos pertinentes, remite la evolución de los animales a lo inorgánico y considera que la naturaleza animal es explicable directamente en términos de lo inorgánico. Parece que se inclina a concebir la evolución de los animales, como constituida por un vasto conjunto de líneas evolutivas que todas siguen un curso común (la scala naturae) y que todas se han originado por generación espontánea (sin intervención de Dios) a partir de lo inorgánico de lo que surge el animal más inferior — - según él un pólipo o un infusorio- y que todas, en el curso de largo tiempo, van ascendiendo, a través de formas cada vez más perfeccionadas, hasta culminar en el hombre. De este modo, Lamarck rechaza, ciertamente, el recurso anticientífico a la creación de las especies, pero lo sustituye por una doble justificación teleológica, no sólo inexplicable al modo científico, en términos de otros procesos reales, inteligibles, sino que él enuncia como un dogma que parece impedir avances paulatinos hacia la solución del esencial problema de la evolución biológica.

Interesa analizar los dos recursos teleológicos de Lamarck inadmisibles para toda conclusión correcta (conforme asevera Kant en la Crítica del juicio). La primera justificación teleológica es el recurso a la generación espontánea - proceso imaginado por Buffon y su colaborador Needham y pronto desmentido experimentalmente con ayuda del microscopio, en 1765, por Lazaro Spallanzani-. Ello no obsta para que constituya una verdad inobjetable que en un remoto tiempo pasado todo indica que hace más de mil millones de años - en las capas superiores de la Tierra no existieran sino moléculas y, por tanto, que necesariamente sobre esa remota base molecular tuvo que producirse el surgimiento de esa leve proliferación superficial que son los seres vivos, y, en último término, los animales; pero este extraordinario resultado ha tenido que producirse en virtud de un proceso, todo indica que escalonado, que cabría explicar en términos de procesos reales concomitantes, en lugar, tiempo y condiciones determinados en los que inicialmente hubieron de intervenir exclusivamente moléculas, procesos potencialmente más o menos inteligibles si - como me inclino a creer - quedan de ellos datos concernientes conservados, por su carácter básico de lo posterior, en el interior de todos los seres vivos actuales. En la enunciación de la generación espontánea como resultado general inherente a lo inorgánico implica conceder a lo inorgánico una tendencia intrínseca inimaginable a constituirse en algo tan cualitativamente distinto como es un determinado primer animal. Teleología que es tan antievolucionista como anticreacionista. La segunda justificación teleológica de Lamarck es atribuir el caracter progresivo de la evolución 
animal —en la que las formas superiores proceden de las inferiores- al instinto propio de los animales de cada estadio evolutivo de su scala naturae de afinar su hábito de conducta de modo que — con la "ayuda" de un tiempo ilimitado- transmute su instinto - sin solución de continuidad y con un cambio mínimo pero dirigido consiguiente de estructuras corporales (la función crea el órgano) - en un instinto progresivo superior de los animales que los lleva a realizarse en el afinamiento de un nuevo hábito, un punto superior, etc.

El monismo científico no se hace cuestión de Dios, sino de ir entendiendo los seres, fenómenos y procesos reales en términos de otros ya esclarecidos; en consecuencia, sustituir la justificación creacionista de la diversidad de formas animales por asertos sin base objetiva, entre ellos justificaciones teleológicas, no es propio del monismo básico de la ciencia. No se puede avanzar en el conocimiento de la Naturaleza sino explicando los procesos reales en términos de otros previamente justificados de este mismo modo, y dilatando así, paso a paso, la comprensión de la coherencia de la realidad, única base de la conquista lenta y paulatina de la verdad científica. De este modo, hay que buscar las soluciones a los problemas científicos planteándolos en términos de otros procesos de la realidad ya mejor o peor entendidos en el sistema de la ciencia. Pues bien, Darwin, procediendo con esa prudencia científica, consigue una interpretación de la evolución que supone un gran avance sobre la de Lamarck, de la que recoge todo lo positivo, a saber: a) el hecho de la evolución animal misma; b) el hecho de que la evolución de cada especie animal se produzca por cambios dirigidos pero ínfimos (Lamarck insiste en que el tiempo es un factor esencial de la evolución animal ${ }^{6}$;) y c) el aserto de que en ella corresponda un papel importante a lo que evoluciona, los animales mismos. Pero corrigiendo su interpretación teleológica y buscando indicios objetivos. En efecto, Darwin, por una parte, indujo que toda especie ha tenido que surgir de la bifurcación de otra ancestral (como culminación del progreso de ésta, cumplido por selección natural); esta inducción se

- Claro que considerar el tiempo, como uno de los factores de la evolución de las especies es un modo impreciso de hablar. El evolucionista tiene que descubrir la naturaleza de los procesos ambientales concretos, coherentes con los individuos de cada especie, que determinan su cambio evolutivo dirigido, fijo. Como la causa de cualquier otro proceso, la causa de la evolución de cada especie opera en un marco espacio-temporal, marco cuyo conocimiento puede ayudar al investigador a establecer la naturaleza concreta de esta causa. Aquí sólo cabe señalar que desde la especiación de que surge una especie hasta la culminación de su evolución en la diferenciación de ella en las dos filiales suelen mediar cientos de miles y hasta millones de generaciones.

Ahora bien, en este sentido circunstancial del tempo de cambio de una especie, Lamarck se adapta a los hechos y no los genetistas que busquen en variaciones de los genomas individuales de pequeñas poblaciones de una especie la causa del surgimiento de una nueva especie, lo que constituye una recaída implícita en el creacionismo que culminó en la definición de una especie como "un monstruo lleno de porvenir", definición — desnuda de toda reserva-que es la correspondiente a la aseveración igualmente creacionista de que en el genoma de su cigoto está el programa del despliegue ontogenético del animal. 
basa en la diferenciación de razas en todas las especies domésticas. De su teoría de la especiación, Darwin sacó la conclusión correcta de que la evolución animal es un proceso que afecta simultáneamente a los animales de todas las especies y en el que, de tiempo en tiempo, los de algunas se diferencian en dos con lo que, con el curso del tiempo $-\mathrm{y}$ aún desquitando las que se han extinguido-, ha ido aumentando aceleradamente el número de especies existentes; esta conclusión (que especifica la diferenciación de las especies como resultado de un proceso potencialmente inteligible) es confirmada por, da cuenta de, los datos de anatomía y embriología comparadas y, sobre todo, permite diseñar el árbol filogénico de las especies sobre la base objetiva del sistema natural de las especies, en el que los criterios de clasificación se subordinan y que se fue estableciendo como resultado de la labor de muy numerosos zoólogos a lo largo de un siglo; a Darwin, además, proyectando su conclusión hacia el pasado, se le impuso que, al remontarse en él, el número de especies ha tenido que ser cada vez menor, lo que le lleva a postular que "todas las especies animales proceden de contado número de especies, tal vez de una sola", esto es, el origen monofilético de todos los animales. Se entiende, por otra parte, que la poderosa imaginación creadora de Darwin, disciplinada a basarse siempre en datos coherentes que permitan soluciones objetivas, se limitara a enunciar el origen monofilético de los animales, pero sin plantearse siquiera cómo pudiera producirse, para lo que carecía de datos concernientes. Hay que tener en cuenta que la definición por Virchow de la célula como unidad de ser vivo es rigurosamente coetánea de la publicación de El origen de las especies (1859) y hoy parece inobjetable que el origen del primer animal ha tenido que ser un resultado culminante de toda una etapa previa de la evolución biológica, la de la evolución celular, cuyo desentrañamiento exige, a su vez, entender la naturaleza de la célula por el proceso de origen de las células, en gran verosimilitud también monofilético, problemas todos con los que está en trance de enfrentarse la biología al siglo y medio de aparecer la obra señera de Darwin. Y, todavía, habrá de hacerlo con la ayuda de su selección natural. (Permítaseme señalar que la gran riqueza de datos empíricos sin interpretar nos ha permitido, en un intenso trabajo de ocho años, desarrollar un primer modelo científico (esto es, conforme con todos los datos disponibles y sin otro modelo alternativo) del proceso de origen de la primera célula, a partir de una determinada asociación de proteínas globulares, proceso en el que ésta fue elevándose a grados crecientes de unitariedad - por una sucesión inteligible de ventajas selectivas - hasta culminar en la primera célula (en el primer foco de acción y experiencia de los seres vivos del segundo nivel de integración); este modelo ha permitido: a) definir la célula por lo que todas las células tienen en común, a saber, los campos físicos unitarios que explican su realización en 


\section{FAUSTINO CORDÓN}

acción y experiencia sobre los ambientes celulares ${ }^{7}$; y b), tras otros cinco años de trabajo, entender la función del metabolismo celular en la acción y experiencia celular propia de lo que consideramos el primer tipo de célula, dar un modelo verosímil de cómo esta célula gobierna su metabolismo, y el de cómo se produjo el despliegue del metabolismo celular inicial ${ }^{8}$. Claro que estos resultados no hubieran sido posibles si, antes, mediante un trabajo continuado de varios años ${ }^{9}$, no se hubiera abierto el acceso evolutivo indispensable hacia el nivel celular, a saber, puntualizando la existencia de seres vivos del primer nivel, su naturaleza y una noción de su proceso evolutivo desde su origen hasta la asociación de ellos ancestral de la primera célula; de estos seres vivos - constituyentes del soma de todas y cada una de las célulashay numerosísimos datos concretos bien establecidos por la llamada biología molecular (designación que ya constituye una contradictio in adjecto), de valor inestimable para entender $a b$ origine la célula pero que se consideran con un reduccionismo a lo molecular generalmente aceptado, muy extraviado y que fue necesario superar; entre la molécula y la célula hay una diferencia abismal de orden de estructura y de tamaño que fueron determinadas por las dos primeras etapas de evolución biológica (la molecular, la basibiónica) sin cuya consideración objetiva no caben sin justificaciones teleológicas de la ontogénesis y filogénesis celular y, a mayor abundamiento, de las del animal cuya explicación se remite a una secuencia de moléculas (los ácidos nucleicos) que se consideran como el programa per se que se cumple - sin saber por quien ni cómo- en cada desarrollo embrionario.)

\section{LAMARCK Y KANT VISTOS POR HAECKEL.}

El pensamiento evolucionista expuesto por Lamarck, en 1809, en su Filosofía zoológica (que hoy probablemente habría titulado teoría zoológica) no se difunde entre sus contemporáneos y permanece prácticamente desconocido al producirse su muerte en 1829. Ahora bien, Darwin, en El origen de las especies (1859), lo considera precursor de su obra y adopta los conceptos lamarckianos positivos, si bien, como se ha señalado, el naturalista inglés eleva la teoría de la evolución de los animales a un nivel superior al de la teoría de Lamarck, cuyas diferencias con su interpretación Darwin no se detiene a analizar. Darwin, como es de conocimiento general, consid-

7 Cordón, F. (1990), Tratado evolucionista de biología. Parte Segunda, «Origen, naturaleza y evolución de la célula», Madrid, Aguilar, vol.I.

8 Ibidem, parte segunda, vol. II

9 Cordón, F. (1978), Tratado evolucionista de biología. Parte Primera «Origen, naturaleza y evolución del basibión y sus asociaciones» (1978), Primera edición, bajo el título La alimentación base de la biología evolucionista, agotada), $2^{\mathrm{a}}$ edición en prensa. 
era que el motor de la evolución de toda especie es la selección natural, como progenitores, ejercida por el "medio-ambiente", de los individuos más aptos; interpretación aún imprecisa pero no teleológica. Por otra parte, considera que toda especie se origina de la bifurcación de otra y evolutivamente culmina en su propia bifurcación, lo que le sugiere que el número de especies ha crecido con el tiempo, y por tanto, ha disminuido al remontarnos en el pasado, lo que le sugiere el posible origen monofilético de los animales; todo lo cual nos lleva a vislumbrar la evolución animal como un proceso de evolución conjunta de sus especies y, además, a plantear, en términos concretos, el origen de cada especie (cuya resolución permitiría entender por su origen la especie correspondiente) y el origen del primer animal (cuya evolución permitiría entender por su origen la naturaleza común de los animales).

Alertado por Darwin, Ernst Haeckel (1834-1919), uno de los primeros darwinistas destacados, en su monumental Historia de la creación de los seres organizados $(1867)^{10}$ hizo una detenida exposición, que constituye un encendido panegírico del pensamiento de Lamarck, al que considera un hito memorable de la historia de la biología. Ello contribuye a rescatar del olvido, justamente, las grandes intuiciones evolucionistas de Lamarck que, hasta 1900, pasan a tener predicamento. Destaca Haeckel, con razón, las aportaciones positivas de Lamarck que recoge Darwin en el suyo, pero no sólo pasa por alto la superación cualitativa de éste sobre la de Lamarck (en este contexto se limita a señalar que Darwin aporta el concepto de la selección natural), sino que no percibe dos graves errores de la interpretación lamarckiana a los que Haeckel mismo se adscribe y a los que conviene insistir porque constituyen prejuicios arraigados que rebrotan, hasta hoy, inspirando las más diversas interpretaciones biológicas. Se trata, por una parte, del recurso a la teleología, y, por la otra, del reduccionismo de la interpretación de seres, fenómenos y procesos de un nivel a lo que se conoce de un nivel de integración inferior (Haeckel, en concreto todo lo reduce a mecanismos).

Con respecto a la teleología la posición de Haeckel es notablemente ambigua. Por ejemplo, al ocuparse de Kant, en la Historia de la creación de los seres organizados, pondera, desde una perspectiva evolucionista, el alcance de la cosmología kantiana a la que considera una teoría admirable que no sólo concuerda con los datos observados, sino que se adelanta cuarenta años a la de Laplace y constituye "en geología e inorganología la vertiente que corresponde a lo que, en biología y antropología, significa la teoría de Lamarck". Ahora bien Haeckel, aunque conviene en que la gran fuerza del kantismo reside en su enfoque monista de la realidad y admite que en la Crítica del juicio Kant parece formular el gran principio de la doctrina ge-

10 En esta obra, Haeckel expone, por primera vez, su teoría de la recapitulación de la filogénesis de todo animal en su ontogénesis, ley con un fondo básico de verdad pero que exige una revisión profunda, desde la comprensión $a b$ origine del primer animal. 


\section{FAUSTINO CORDÓN}

nealógica ${ }^{11}$, en cambio, le reprocha que renuncie al mecanicismo al estudiar los seres vivos, en beneficio de la teleología. Según cita de Haeckel, Kant declara en la Crítica del juicio: "Es absurdo esperar que un nuevo Newton venga a explicarnos la producción de una brizna de yerba a la que no haya presidido ningún designio; pues es una visión que hay que negar a los hombres". Curiosamente, comenta con escándalo Haeckel, la Crítica niega, en suma, a la razón humana el poder de explicar mecánicamente los fenómenos de la naturaleza orgánica, no dándole esa competencia sino sobre el terreno de la naturaleza inorgánica. Pero basta acercarse un poco a Kant para entender lo riguroso, lo justicado de su postura. Considera que el problema de los cuerpos orgánicos, cuyas partes son recíprocamente medio y fin unas de otras, no puede resolverse en términos meramente mecánicos porque implicaría una excesiva coincidencia suponer que se hayan podido producir por el juego ordinario de fuerzas mecánicas. Ahora bien, si recusamos, como su origen, el mecanismo y recurrimos al de la Naturzweck (propósito de la Naturaleza) implicamos que haya cosas a las que deba considerarse resultado de un designio sobrenatural y, así, pasar de lo sensible a lo suprasensible de un modo explícitamente prohibido por la Crítica de la razón pura. Ante esta dificultad Kant recurre, de nuevo, al juicio reflexivo. Piensa que no podemos evitar el juicio teleológico para dar cuenta de los seres vivos pero tomándolo como una solución transitoria. Debemos, dice, considerar los organismos como si fueran resultado de un designio, que no es lo mismo que decir que son producidos deliberadamente.

En contra del materialismo-vulgar de Haeckel, el monismo ha de intentar, al modo científico, introducir el "espíritu" (los contenidos de conciencia en que se realizan los seres vivos) en el Universo: procurar dar cuenta del "espíritu" en términos del proceso de toda la realidad. El monismo-mecanicista (otra buena contradictio in adjecto) procura expulsar fuera de la realidad las manifestaciones del "espíritu" como si, contra toda evidencia, no existieran ${ }^{12}$. De hecho, por expulsarlas fuera, estas manifestaciones no dejan de arraigar en una u otra forma de creacionismo. Parece que el monismo mecanicista, el materialismo vulgar, es un monismo sin convicción, temeroso. Para Lamarck (1809) el instinto, al que considera el agente de la evolución, radica en el interior de cada animal impulsándolo, como vector de la causa final, teleológicamente, a ascender en la scala naturae. Ciertamente el "espíritu" aparece operando pero es considerado, ininteligiblemente, como resultado

\footnotetext{
$11 \mathrm{Al}$ considerar la semejanza entre las formas orgánicas revelada por la anatomía comparada, Kant se encaminaba hacia un prototipo común del que ellas derivan. (Raíz kantiana del pensamiento biológico de Goethe, de von Baer y de Geoffroy Saint-Hilaire.)

12 Con igual razón, no sujetarse al mecanicismo manejado por el hombre, haría recusar como inexistentes todas las manifestaciones de las reacciones químicas, de los procesos intratómicos, etc.
} 


\section{EL PENSAMIENTO DE LAMARCK EN SU CONTEXTO HISTÓRICO}

y como agente de fuerzas mecánicas, a las que se remite también la reproducción de los animales, supuestamente gobernada por sólo leyes mecánicas.

Darwin (1859) remite el proceso de la evolución al ser vivo al resto de la realidad, a saber, a un medio-ambiente que, implícitamente, ha de operar de modo correspondiente a cómo lo hace el hombre en su selección de razas domésticas (en la que, como en todo lo que realiza, éste aplica su capacidad de tomar noticia y de decisión que nos son propias). Asimismo para Darwin lo que se selecciona, como progenitores, son animales con la conveniente aptitud de adaptarse a las condiciones impuestas por el medio-ambiente selector. Darwin, sin duda, atiende a los datos reales que le competen con una mirada directa, no impedida por gazmoñería mecanicista; ve a los animales vivir, aunque esté fuera del alcance de su época plantearse qué sea vivir en términos del proceso conjunto de lo real.

Sin embargo, el entusiasta darwinista Haeckel, considera, en 1867, como ciencia la reducción de todos los procesos reales a monismo-mecanicismo, al menos, a profesión de fe monistamecanicista. Habría de admitir, contra toda evidencia, que la misma toma de noticia de su propia circunstancia o no existe o es una mera manifestación de fuerzas mecánicas (las que operan en las máquinas que hace el hombre), sin que le mereciese la pena explicar cómo se produzca ello.

Por último Kant -al que culpa Haeckel de infidelidad al monismo-mecanicismo-, al considerar, hacia 1790, los seres vivos, parece mantenerse como un monista riguroso al aseverar que los animales no pueden ser resultado de meras fuerzas mecánicas (inteligibles por el hombre en cuanto productor de mecanismos que se basan en leyes mecánicas por él descubiertas), sino que "parece que fueran resultado de un designio", ya que es inconcebible que se realice por azar el complejo y perfecto coajuste que se da entre las partes - los órganos- de los animales y de las plantas de todas las especies. Explícitamente a Kant no le satisface este "como sí" para justificar lo que él tiende ya a sospechar resultado de un proceso evolutivo ${ }^{13}$, y lo considera un recurso propedeútico, que espera solución, de cómo esa perfecta coordinación organísmica se produjo (y sigue produciéndose) en términos del proceso de la realidad.

Tengo la convicción de que la capacidad de acción y experiencia (ésta con su cuánto de conciencia y libertad) sobre sus ambientes específicos que caracteriza a los seres vivos, debidamente enfocada puede permitir la ideación de modelos científi-

\footnotetext{
13 Los datos de anatomía comparada ya hacen intuir a Kant la comunidad de estructuras de las distintas especies, comunidad que Geoffroy Saint-Hilaire (en 1830) intentó inútilmente concretar, con descrédito para su certera sospecha, frente al anatomista comparado creacionista Cuvier.
} 


\section{FAUSTINO CORDÓN}

$\cos ^{14}$, gracias a la gran riqueza de datos acumulada por la biología experimental, capaces de dar cuenta de la naturaleza física de la acción y experiencia (ésta, insistimos, con su cuánto de conciencia y libertad) de los animales remitiéndola a la comprensión del proceso conjunto de la evolución de células y de asociaciones de células, comprensión que, a su vez, exige el conocimiento, $a b$ origine, de la primera célula, y, a partir de él, la comprensión de la naturaleza de la célula. Asimismo, el conocimiento monista de la célula, lejos de todo mecanicismo, remite al conocimiento de la naturaleza física de la acción y experiencia de seres vivos del nivel directamente supramolecular y subcelular, conocimiento ya perfectamente inteligible, que, a su vez, remite a procesos del nivel molecular, esto es, por fin, a lo "inorgánico". En definitiva, conforme con un monismo riguroso, se va adquiriendo una primera interpretación de cómo, a partir del nivel superior de lo inorgánico, se pudo producir (conforme a lo que atestiguan datos comunes del interior de las células actuales) el origen monofilético de los seres vivos en la Tierra, con lo que se salva la barrera que parecía irrebasable entre lo inorgánico y lo orgánico, y cómo se inició y se ha ido desplegando la evolución biológica, en la que se suceden etapas (la molecular, la basibiónica ${ }^{15}$, la celular, la animal), cada una de las cuales culmina en el surgimiento de seres vivos de un nivel más de integración de cuyo estudio -hecho posible por el logro de la ciencia experimental de deslindar los niveles de entidades homogéneas-, en cada uno de los cuales (átomos, moléculas, basibiones, células, animales) a la biología evolucionista le corresponde esclarecer un tipo de problemas de naturaleza distinta, a saber, cómo se relacionan entre sí entidades heterogéneas, en concreto, las unidades de cada dos niveles biológicos sucesivos: basibiones y moléculas subordinadas, células y basibiones subordinados y animales y células subordinadas. Estudio en el que, por una parte, en cada etapa evolutiva rigen leyes distintas, lo que veta toda extrapolación viciosa (toda integración pseudomonista) y, por otra parte, no puede abordarse con fruto la evolución de una etapa sin el conocimiento indispensable de la etapa inmediata anterior, como básica que es de la considerada.

Para terminar señalemos dos consecuencias monistas deducidas del enfoque actual de la evolución biológica. La primera es que el proceso de la evolución

\footnotetext{
14 Consideramos que un modelo merece la calificación de científico en tanto que a) satisfaga todos los datos concernientes y b) no exista otro modelo alternativo.

15 Entendemos por basibión -ser vivo básico- el ser vivo del primer nivel de integración, el directamente supramolecular e infracelular, dotado de un modo de acción y experiencia bien establecido propio de su nivel y de una estructura netamente supramolelcular (la propia de las proteínas globulares). Véase CORDón, F. (1994), «Las proteínas globulares, su estructura y función supramoleculares», Mundo Científico, $\mathrm{n}^{\circ} 143$ y 144 .
} 


\section{EL PENSAMIENTO DE LAMARCK EN SU CONTEXTO HISTÓRICO}

biológica es complementario del despliegue de la capacidad de tomar noticia del ser vivo que evoluciona (capacidad, insistamos, potencialmente inteligible, al modo monista, en términos del proceso del nivel de la realidad que corresponda): la conciencia es resultado de la evolución y, a la inversa, la evolución lo es de la conciencia de los seres vivos de las etapas de evolución correspondientes. Y parece verosímil que esta aseveración pueda extrapolarse a la evolución de los niveles de lo inorgánico, como explicación de que la materia universal no sea ni absolutamente determinada ni desordenada, sino - eterna y sin límites- experimentable en cuanto experimentante y viceversa.

En segundo lugar, las microestructuras comunes a los seres vivos de cada nivel (que preocuparon a Kant, a Goethe, a von Baer y, en particular, a Geoffroy SaintHilaire) creo que existen y son rastreables; opino que han de estar constituidas por conjuntos de series elementales de seres vivos somáticos del nivel inmediato inferior, series cada una de las cuales consta de un número fijo de tales seres vivos, cada uno especializado en cooperar en la producción de uno de los campos físicos unitarios en cuya coordinación el ser vivo de nuevo nivel se realiza en pulsaciones de acción y experiencia. Este resultado, en cuanto definidor del nuevo ser vivo, ha de conservarse como condición básica de toda la evolución (de toda su diferenciación progresiva en órganos) de los seres vivos de dicho nivel. 